\title{
Simulación del Depósito de Escarcha en Superficies Frías
}

Jorge A. Gatica ${ }^{1}$, Vicente A. Pita ${ }^{1}$ y Nisio de C. Lobo ${ }^{2}$

(1) Universidad del Bío-Bío, Departamento de Ingeniería Mecánica, Avenida Collao 1202, Concepción-Chile (e-mail: jgatica@ubiobio.cl; vpita@ubiobio.cl )

(2) Universidade Federal do Rio de Janeiro, Programa de Engenharia Mecânica,

Avda. Tromposky 1202, Rio de Janeiro-Brasil (e-mail: nisio@serv.com.ufrj.br)

\section{Resumen}

Se presenta un código computacional que simula la formación y crecimiento de escarcha sobre una placa mantenida a baja temperatura, por ablimación del vapor de agua de una corriente de aire húmedo que fluye sobre ella. La modelación se basa en ecuaciones de conservación de masa, energía, cantidad de movimiento y especies aplicadas a la escarcha, mezcla aire-vapor e interfase aire-escarcha. El sistema de ecuaciones resulta no lineal acoplado por la fracción volumétrica del hielo, que es calculada iterativamente. El modelo es validado frente a datos experimentales, permitiendo reproducir la evolución de propiedades termofísicas de la escarcha formada y predecir comportamientos para otras condiciones de operación. Los resultados revelan mejor concordancia con datos experimentales que la obtenida por otros autores, y levemente inferior a la del modelo de sobresaturación.

Palabras clave: crecimiento de escarcha, código computacional, simulador, propiedades termofísicas

\section{Simulation of Frost Deposit in Cold Surfaces}

\begin{abstract}
The development of a computational code that simulates the formation and frost growth on a cool plate, for the vapor-water ablimation of a humid air current that flows on it, is presented. The numeric modeling is based on the conservation equations of mass, energy, momentum and species applied to the frost, air-vapor mixtures and air-frost interface. The system of equations is not linear and is coupled by the ice volumetric fraction which is calculated by iteration. The model is validated by comparing the numerical results with experimental data, reproducing the thermophysical properties evolution of the frost formed and predicting the behavior for other operating conditions. The results reveal better agreement with experimental data that those obtained by other authors, and slightly inferior to that of the supersaturation model.
\end{abstract}

Keywords: frost growth, computational code, simulator, thermophysical properties, modeling 


\section{INTRODUCCIÓN}

La formación de escarcha en la superficie externa de intercambiadores de calor en contacto con aire, operando en condiciones de temperatura bajo cero grados, es un fenómeno frecuente en sistemas de refrigeración industrial y doméstica. Este fenómeno es indeseable pues afecta la transferencia de calor y agrava la pérdida de carga por el lado del aire, disminuyendo la capacidad de refrigeración.

La necesidad de periodos de detención del sistema de refrigeración para la remoción de la capa de escarcha, excesivamente espesa en las aletas de evaporadores, llega a comprometer fuertemente la operación de tales equipamientos. Por lo anterior, la comprensión física y el análisis del fenómeno de transferencia de calor y masa en la capa de escarcha formada, puede facilitar el diseño de evaporadores de alta eficiencia térmica.

En el desarrollo de este artículo fueron consultados trabajos analítico-experimentales de O’Neal y Tree (1984), quienes caracterizan los efectos de parámetros: humedad y temperatura del aire, temperatura de la placa, número de Reynolds, etc., en la formación y crecimiento del hielo sobre una superficie fría. De sus resultados experimentales concluyen que aumenta el crecimiento de la escarcha ante incrementos del número de Reynolds mientras este sea inferior a 15900, valores superiores no ejercen influencia. Tales conclusiones para Reynolds menores a 15900 son respaldadas por Sahin (1994), con números de Reynolds de 2400, 3700 y 4500 y por estos autores para Reynolds entre 5000 y 15000 . Conclusiones para valores superiores también son respaldadas en este trabajo para Reynolds 20000, 25000 y 30000.

O'Neal y Tree (1985), realizan una interesante revisión de correlaciones para la conductividad térmica de la capa de escarcha. Muestran que cada correlación fue desarrollada para un cierto rango de temperaturas. La forma de los cristales de hielo es dependiente de esta propiedad.

Ha habido varios esfuerzos empíricos y semiempíricos para modelar el crecimiento de la escarcha, que pueden ser resumidos en Padki et al. (1989). Un modelo físico de Tao et al. (1993), acopla calor y difusión de humedad para modelar la escarcha como un medio poroso y compara los resultados de su simulación con correlaciones experimentales de Mao et al. (1992), este último mostró que la concentración de masa y el espesor de la escarcha varían con el tiempo y el espacio y determinó, similar a Le Gall et al. (1997), correlaciones empíricas para la formación de escarcha.

Tao et al. (1993), introducen un coeficiente de difusión efectiva modificado para el vapor de agua en el interior de la capa de escarcha y asume algunas condiciones de contorno no basadas en datos físicos. Su aporte fue tomado en cuenta para la estimación del coeficiente de difusión efectivo en este trabajo. Le Gall et al. (1997), modifica el modelo de Tao eliminando una ecuación y la necesidad de dos condiciones de contorno y modificó el coeficiente de difusión efectiva del vapor de agua usando una función continua, logrando razonable concordancia entre sus simulaciones y datos de Mao et al. (1992). Sin embargo su propuesta, de acuerdo con la línea de Auracher (1972), no fue aprovechada.

Lee et al. (1997), desarrollan un modelo analítico para la formulación de la escarcha sobre una superficie fría, considerando difusión molecular del agua y generación de calor producto del proceso inverso a la sublimación, en adelante ablimación, del vapor de agua en la capa de escarcha. Ellos asumen que el vapor de agua difunde en dirección normal a la placa enfriada. Su modelo, que desacopla la transferencia de calor y masa, predice el comportamiento de formación de escarcha y muestra razonable concordancia con su base de datos experimental. Mao et al. (1999), presentan un trabajo experimental de crecimiento de hielo sobre una superficie plana, a baja temperatura, sometida a un congelamiento turbulento, de flujo paralelo del aire húmedo, en condiciones de operación típicas de muchos congeladores comerciales. Obtienen una correlación empírica para el coeficiente de transferencia de masa durante el crecimiento de la escarcha; sin recurrir a la analogía entre transferencia de calor y masa típicamente utilizada. Un modelo para predecir el crecimiento de la escarcha, de Chen et al. (2000), muestra una fuerte caída en el rendimiento de un conjunto ventilador-serpentín aletado, ante aumentos de espesor de la escarcha formada. Yun et al. (2002), modela físicamente el crecimiento de la escarcha y sus propiedades. 
En dos interesantes trabajos relativos a la tasa de crecimiento de escarcha, Na y Webb (2004a; 2004b), demuestran experimental y analíticamente la necesidad de sobresaturación en la superficie de la escarcha y aplican este criterio para predecir su crecimiento, obteniendo simulaciones muy de acuerdo con sus resultados experimentales. Lee y Ro (2005), modelan, a semejanza de Na y Weeb (2004a; 2004b), asumiendo concentración agua-vapor en superficie de la escarcha en sobresaturación, para corregir inconsistencias del modelo de saturación. Un modelo matemático k- $\varepsilon$ para flujo turbulento prueba que este flujo (Yang et al. 2006), a diferencia del laminar, influye en el crecimiento de la escarcha. Aumentos de velocidad del aire, influyen inversamente para estos flujos en la transferencia de masa. A menores temperaturas del aire mayor espesor de escarcha. Un trabajo experimental de Qu et al. (2006), analiza situaciones criogénicas. Kim et al. (2008), proponen una correlación adimensional para las propiedades de la escarcha sobre una superficie cilíndrica fría.

En este trabajo, como en el de muchos otros investigadores; Le Gall et al. (1997), Lee et al. (1997) y Tao et al. (1993), se asume que el vapor de agua es saturado en la superficie de la escarcha. Si bien el modelo de sobresaturación es más riguroso, los resultados y validaciones que son obtenidos por los autores de este trabajo, son bastante razonables.

Resultados experimentales de O'Neal y Tree (1984), Sahin (1994), Chen et al.(1999) y Na y Webb (2004b), son considerados en este artículo para validar el modelo propuesto.

En este estudio se desarrolla un simulador numérico que, a partir del conocimiento del estado termodinámico del aire y su velocidad, permite analizar el comportamiento de la escarcha formada a través de un modelo físico matemático, que supone que la concentración de vapor de agua en la superficie de la escarcha es saturada, (modelo de saturación). Su finalidad es permitir una mayor comprensión del fenómeno físico involucrado. El modelo es verificado comparando los resultados con datos experimentales de distintos investigadores. El modelo permite predecir variaciones temporales y espaciales de las propiedades termofísicas de la escarcha formada.

\section{MODELACION FISICA}

Cuando una placa limpia, a temperatura bajo cero grados es expuesta a un flujo de aire húmedo, la formación y crecimiento de escarcha sobre su superficie, puede resumirse en tres periodos:

Periodo de crecimiento del cristal (periodo inicial breve): Se caracteriza por la condensación y congelamiento de pequeñas gotas de agua. Sobre el núcleo de hielo formado surgen cristales de hielo, que crecen verticalmente a tasa casi constante. Por sus características no fue considerado en el presente estudio, siendo reemplazado por el establecimiento de las condiciones iniciales del segundo período.

Período de crecimiento de la escarcha: Este periodo y el siguiente son los más relevantes en la evolución de la capa de escarcha. Durante ambos periodos, ésta presenta un aspecto más uniforme debido a la ramificación e interconexión de los cristales de hielo. La capa de escarcha se torna un material poroso homogéneo, hecho de una matriz de hielo sólido y poros llenos de aire húmedo. La transferencia de masa en la capa de escarcha lleva al proceso de crecimiento y densificación del depósito poroso. Tudor et al. (2003), consigue controlar la forma y tasa de crecimiento de los cristales de escarcha, utilizando campos eléctricos establecidos con corriente continua y alterna. Es aplicable la modelación de medios porosos para la capa de escarcha.

Período de crecimiento intenso: Este aparece cuando la temperatura superficial alcanza la temperatura del punto triple del agua debido al aumento de la resistencia térmica del hielo. El vapor de agua condensado en la superficie de la capa de escarcha, forma una película líquida que satura la capa de escarcha, y se congela en las áreas más próximas a la pared fría. Así, ocurre un proceso cíclico de fusión, congelamiento y crecimiento, hasta lograr el equilibrio térmico de la capa de escarcha.

El sistema a analizar, Fig. 1, consiste de una placa plana horizontal, sobre la cual fluye aire húmedo. La placa es isotérmica, con bajo cero grados siempre inferior a la temperatura del aire, esto permite 
que el vapor de agua del aire se deposite, inicialmente sobre la placa en fase líquida (escarcha por condensación) o directo en la forma de cristales de hielo (escarcha por ablimación).

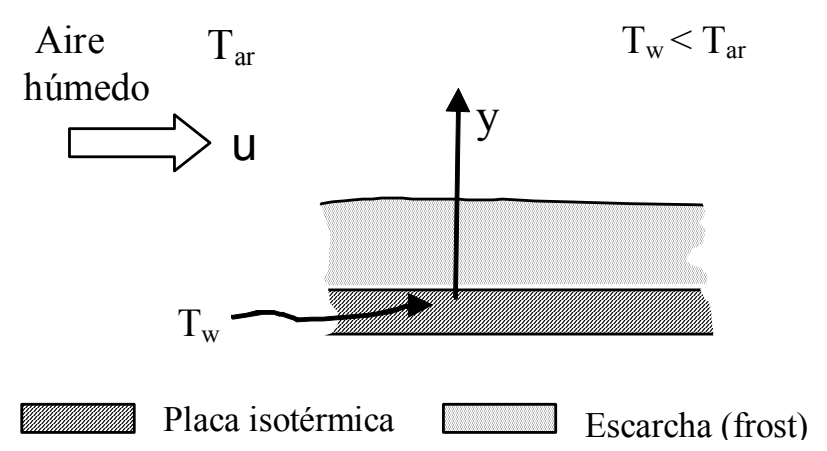

Fig. 1: Esquema del sistema en estudio

Similar a Le Gall et al. (1997), Ismail y Salinas (1999) y Luer y Beer (2000), la escarcha se considera como un medio poroso. En rigor la fase sólida tendría volumen variable en el tiempo, debido a la solidificación del vapor que difunde a través de los poros formados en los espacios de la estructura arbórea de la escarcha producida. La modelación físico-matemática, considera: aire húmedo forzado sobre la escarcha; fluidos incompresibles; propiedades del aire variables en la capa porosa; efectos gravitacionales despreciables; presión total constante de la fase gaseosa; aire húmedo como mezcla de gases ideales y saturado en el interior de los poros; y superficie refrigerada impermeable.

Si se considera un poro, formado en el interior de la estructura arbórea del hielo, próximo a la superficie fría, se observará que quedará deficiente de vapor de agua en el tiempo, a causa de su transformación en hielo en la superficie de dicha estructura. Así, la mayor concentración de vapor de agua en las capas superficiales del depósito de escarcha, se difunde con la finalidad de compensar el desequilibrio provocado por la ablimación. En el caso presente, la difusividad molecular $\mathrm{D}_{\mathrm{AB}}$ del vapor en el aire, no gobierna el proceso difusivo dado que el hielo ofrece una resistencia a la difusión. Una interesante discusión es hecha en Gatica (2003), en relación al factor de resistencia a la difusión $\mu$, lo cual llevó a desestimar la propuesta de Le Gall et al. (1997) y retomar la utilización de los parámetros, $F$ para el interior de la escarcha y $F_{s}$ para la superficie de la capa de escarcha, como fue propuesto en el trabajo de Tao et al. (1993). Una fuerte dependencia en el valor de dichos parámetros, fue verificada en el trabajo de Gatica, generando diversos espesores finales y saltos en la densidad, que aún cuando pueden ser físicamente posibles, resultan incoherentes con el modelo físico matemático estudiado. Se buscó valores de $\mathrm{F}_{\mathrm{s}}$ cuya validez sería avalada por los resultados de evolución del espesor que produjesen.

\section{MODELO MATEMÁTICO}

Se considera un modelo numérico unidimensional transiente, estructurado sobre ecuaciones de conservación de masa y energía, cantidad de movimiento y especies (Gatica, 2003; Ismail y Salinas, 1999 y Le Gall et al., 1997) aplicadas a la escarcha, mezcla aire-vapor y a la interfase aire-escarcha. La escarcha es considerada un medio poroso pseudo-homogéneo, en el cual la transferencia de calor y masa es descrita, por leyes de Fourier y Fick. Estos fenómenos de transporte son acoplados.

Se aplica la técnica del volumen local medio en un volumen de control elemental $V$ donde coexisten $V_{1}$ y $V_{g}$, definiéndose fracciones volumétricas (Ec. 1) y compatibilidad volumétrica (Ec. 2):

$$
\begin{aligned}
& \varepsilon_{\mathrm{g}}=\mathrm{V}_{\mathrm{g}} / \mathrm{V} \quad \mathrm{y} \quad \varepsilon=\mathrm{V}_{\mathrm{l}} / \mathrm{V} \\
& \left(\frac{\partial \varepsilon}{\partial \mathrm{t}}\right)=-\left(\frac{\partial \varepsilon_{\mathrm{g}}}{\partial \mathrm{t}}\right)
\end{aligned}
$$


Ecuaciones de energía y masa

Ecuación de la energía

$\frac{\partial}{\partial t}\left(\rho C_{p} T\right)-\rho_{l} l_{i g} \frac{\partial \varepsilon}{\partial t}=\operatorname{div} k_{\text {eff }} \nabla T$

Ecuación de difusión fase gas-vapor

$\frac{\partial}{\partial t}(1-\varepsilon) \rho_{\mathrm{v}}+\rho_{\mathrm{I}} \frac{\partial \varepsilon}{\partial y}=\operatorname{div}\left[(1-\varepsilon) D_{A B} \nabla \rho_{\mathrm{v}}\right]$

Ecuaciones de las condiciones de contorno

Se supuso que el vapor estaría saturado en todos los poros de la escarcha. Si además se trata como un gas ideal, su densidad $\rho_{\mathrm{v}}$ es sólo función de la temperatura y la ecuación de conservación de masa presentada antes, es en verdad una ecuación para la fracción volumétrica de hielo $\varepsilon$. Los extremos de la escarcha son analizados de forma distinta a los puntos interiores de la misma, pues la expresión de conservación del vapor está alterada en estas zonas.

Así se tiene, junto a la placa fría impenetrable (Ec. 5) y en la interfase escarcha-aire húmedo (Ec. 6):

$$
\begin{aligned}
& \left.D_{\text {eff }} \frac{\partial \rho_{v}}{\partial y}\right|_{y=0}=0 \\
& \left.D_{\text {eff }} \frac{\partial \rho_{v}}{\partial y}\right|_{y=y_{s}}=h_{m}\left(\rho_{v \infty}-\left.\rho_{v}\right|_{y=y_{s}}\right)-\left.\left(\rho \frac{\partial y}{\partial t}\right)\right|_{y=y_{s}}
\end{aligned}
$$

Para la ecuación de la energía las condiciones de contorno e inicial se definen por: (Ec. 7) y (Ec. 8).

$\mathrm{T}=\mathrm{T}_{\mathrm{W}}$

$h_{c v}\left(T_{a r}-\left.T\right|_{y=y_{s}}\right)=\left.k_{\text {eff }} \frac{\partial T}{\partial y}\right|_{y=y_{s}}-\left.I_{i g}\left(\rho \frac{\partial y_{s}}{\partial t}\right)\right|_{y=y_{s}}$

El sistema de ecuaciones obtenido, se cierra considerando las propiedades termofísicas. La fase de cristalización se resuelve, considerando al inicio un espesor reducido de escarcha, con temperatura uniforme igual a la de la placa fría.

\section{Propiedades Termofísicas}

De la literatura disponible (Le Gall et al., 1997; O'Neal y Tree, 1985; Dietenberger, 1983; Ismail y Salinas, 1999; Tao et al., 1993 y Luer y Beer, 2000), se obtiene en unidades SI, las correlaciones para las propiedades del aire seco y del vapor de agua (Ecs. 9 a 14), propiedades térmicas del hielo y de la escarcha (Ecs.15 a 22). Para propiedades del aire húmedo se utiliza ASHRAE (1997).

$k_{a}=-3.381 E-08 T^{2}+9.814 \mathrm{E}-05 T-1.308 E-04 \quad(180 \leq T \leq 600)$

$D_{\text {eff }}=(1-\varepsilon) D_{A B}(1+F)$

OBS: Se emplea $\mathrm{F}$ o $\mathrm{F}_{\mathrm{s}}$ (Ec. 10) para región interior o superficial de la escarcha, respectivamente. 


$$
D_{A B}=1.451 E-04 \frac{T^{1.72}}{p_{a t m}}
$$

$\mathrm{Nu}=0.038 \mathrm{Re}^{0.75} \mathrm{Pr}^{0.33}$

$\mathrm{h}_{\mathrm{cv}}=\mathrm{k}_{\mathrm{a}} \cdot \mathrm{Nu} / \mathrm{L}$

$\mathrm{h}_{\mathrm{m}}=\mathrm{h}_{\mathrm{cv}} /\left(\rho_{\mathrm{a} \infty} \cdot C \mathrm{p}_{\mathrm{a}}\right)$

$k_{1}=630 / T$

$C p_{1}=2109+3.66(T-273.15)$

$\rho_{1}=1 /(0.0010907+1.4635 E-07 T)$

$\mathrm{I}_{\text {ig }}=(-0.047(1.8(\mathrm{~T}-273)+32)+1220) 2322$

$\rho=\varepsilon \rho_{1}+(1-\varepsilon)\left(\rho_{a}+\rho_{v}\right)$

$C p=\left(\varepsilon_{1} \rho_{1} C p_{a}(T)+\left(1-\varepsilon_{1}\right)\left(\rho_{a} C p_{a}+\rho_{v} C p_{v}\right)\right) / \rho$

$\mathrm{k}_{\mathrm{eff}}=\varepsilon \mathrm{k}_{\mathrm{l}}+(1-\varepsilon) \mathrm{k}_{\mathrm{a}}$

$\sigma=\frac{\rho_{1}-\rho}{\rho_{1}-\rho_{p}} \quad$ con, $\rho_{p}=\rho_{v}+\rho_{a}$

\section{Solución Numérica}

Las ecuaciones diferenciales parciales, de condiciones de contorno y constitutivas, conforman un sistema no lineal que es acoplado por la fracción volumétrica del hielo, $\varepsilon$, la cual se calcula iterativamente a través del uso de (Ec. 4). Esta última ecuación diferencial es de primer orden, lo que provoca una sensible rigidez cuando se intenta obtener la solución numérica en su forma conservativa y crea aproximaciones por diferencias finitas con la misma propiedad.

Se utilizó un esquema implícito para las dos ecuaciones principales, pues fue generada una matriz tridiagonal para el sistema de ecuaciones algebraicas por la discretización de la ecuación de la energía, requiriendo aproximación de primer orden en el tiempo y segundo orden en el espacio (en el contorno se usó aproximación de primer orden en el espacio). Para la fracción volumétrica usada, el sistema generado es no-lineal y se resuelve con la subrutina DNEQNF del paquete IMSL. El tratamiento de las no-linealidades, debido a la dependencia existente entre las propiedades y la temperatura, se hace con un atraso en su cálculo con posterior corrección iterativa, hasta satisfacer una tolerancia dada. Asimismo se utilizó una sub-relajación para garantizar una mejor estabilidad al obtener la solución.

Este problema sufre expansión en su dominio a cada paso en el tiempo, debido al aumento de espesor de la escarcha. Este incremento generaba una redefinición de la malla a cada instante, así los valores de las propiedades en los nuevos nodos se logran por interpolación con los valores anteriores obtenidos. En Fig. 2 puede verse un diagrama de bloques del programa desarrollado.

La inicialización parte del arbitraje de un espesor inicial de escarcha con una fracción volumétrica y temperatura definidas, ambas uniformes. 

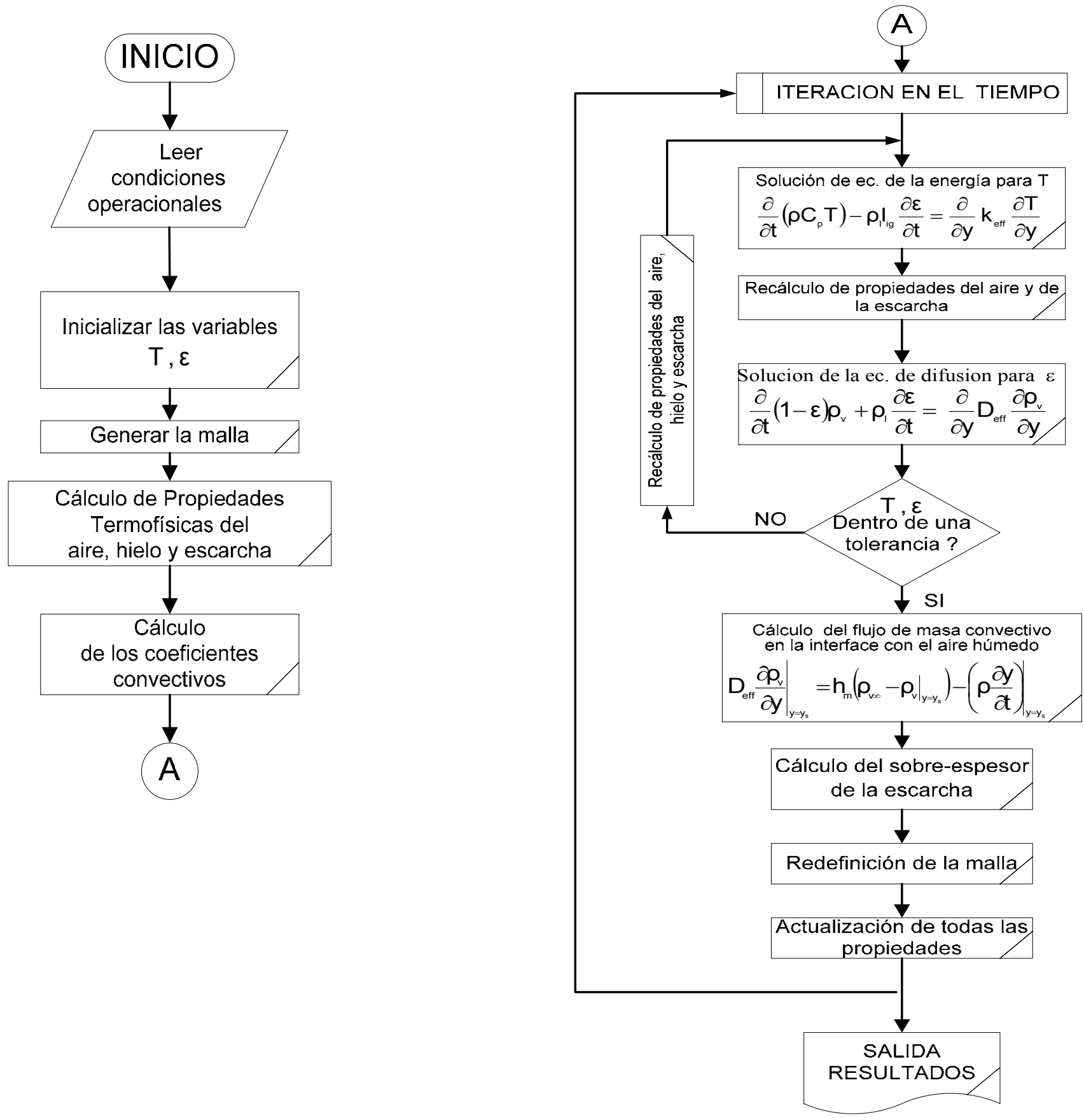

Fig. 2: Algoritmo de la simulación

\section{Análisis Previos}

Definición de la malla: Inicialmente se busca optimizar la malla utilizada en la simulación numérica, en el espacio y en el tiempo. Este estudio consideró la variación de espesor de la capa de escarcha durante 6 horas de simulación.

En Figuras 3 y $3 a$, se busca optimizar el paso de tiempo $\Delta t \circ(\mathrm{Pt})$ y se observa que existe alta insensibilidad en relación al espesor, incluso para Reynolds diferentes. Similar situación muestran las Figs. 4 y 4 a para la optimización de la malla M. Alguna aclaración se obtiene si se observa las Figs. $3 \mathrm{~b}$ y $4 \mathrm{~b}$, que amplían el comportamiento para algún número de Reynolds en cada caso. Se observa mayor convergencia entre 1 y $0.5 \mathrm{~s}$ de paso de tiempo y para mallas entre 10 y 40 . Razonablemente se escoge $\mathrm{Pt}=0.5 \mathrm{~s}$ y $\mathrm{M}=30$. Resultados posteriores avalan esta elección. 


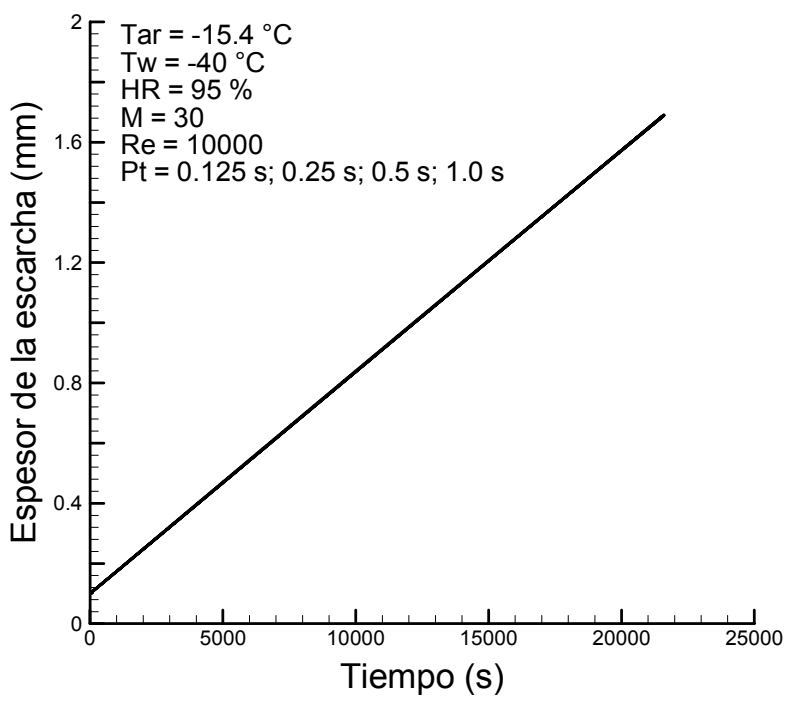

Fig. 3: Optimización del paso de tiempo $\Delta \mathrm{t}$

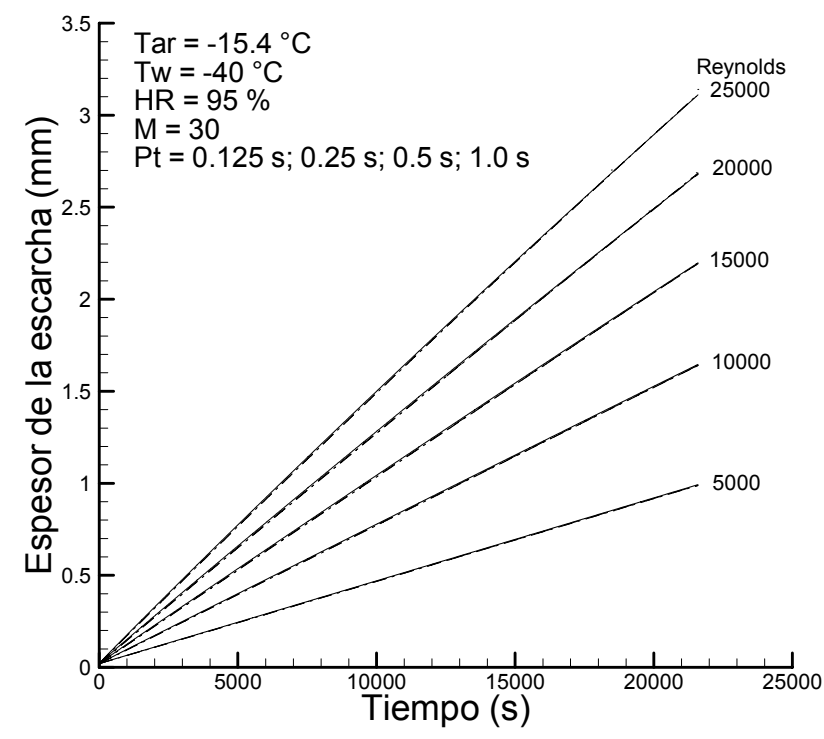

Fig. 3a: Pasos de tiempo vs. Reynolds

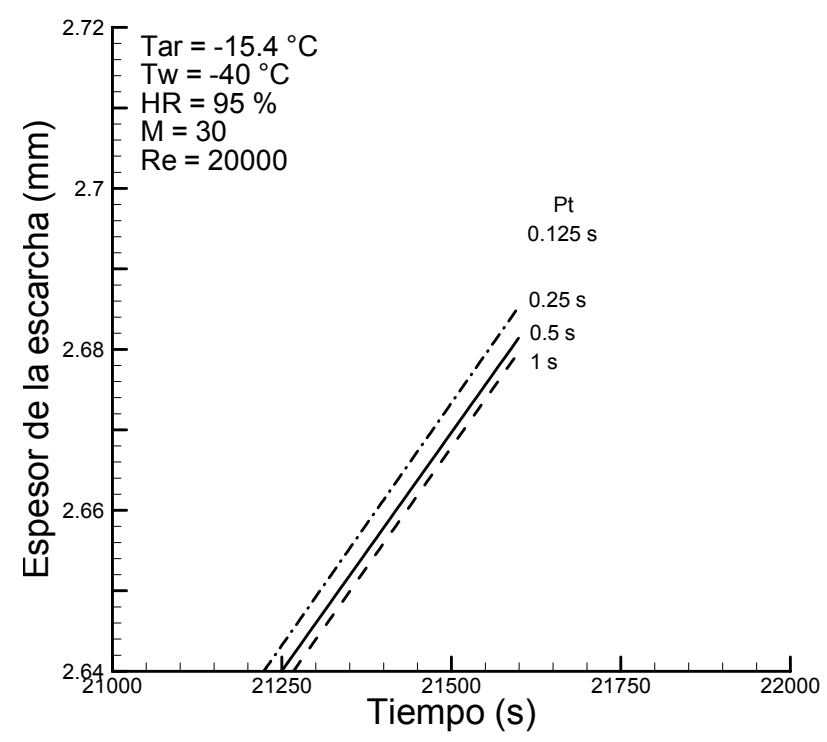

Fig. 3b: Ampliación para cierto Reynolds

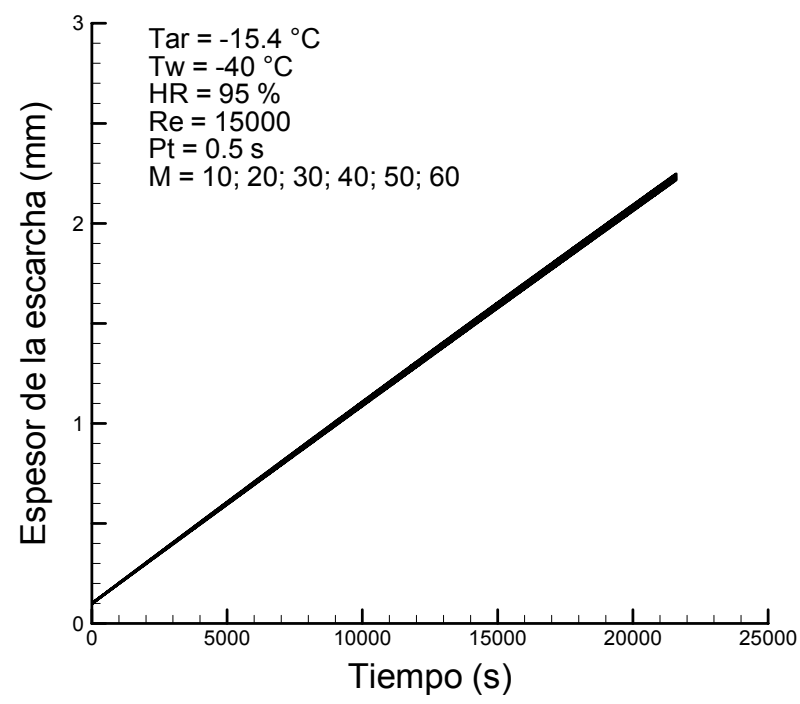

Fig. 4: Optimización del número de puntos

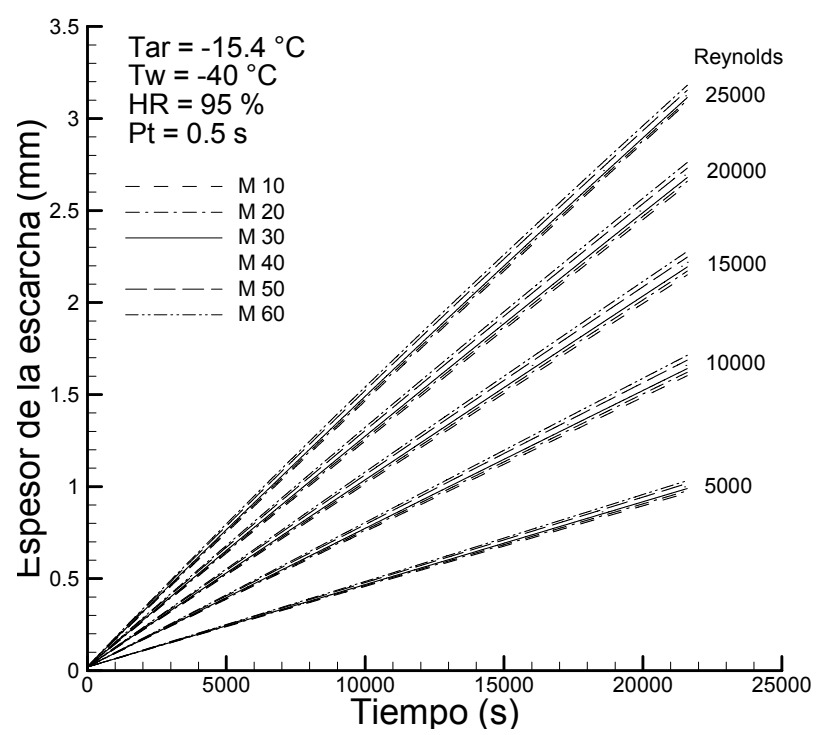

Fig. 4a: Tamaño malla vs. Reynolds

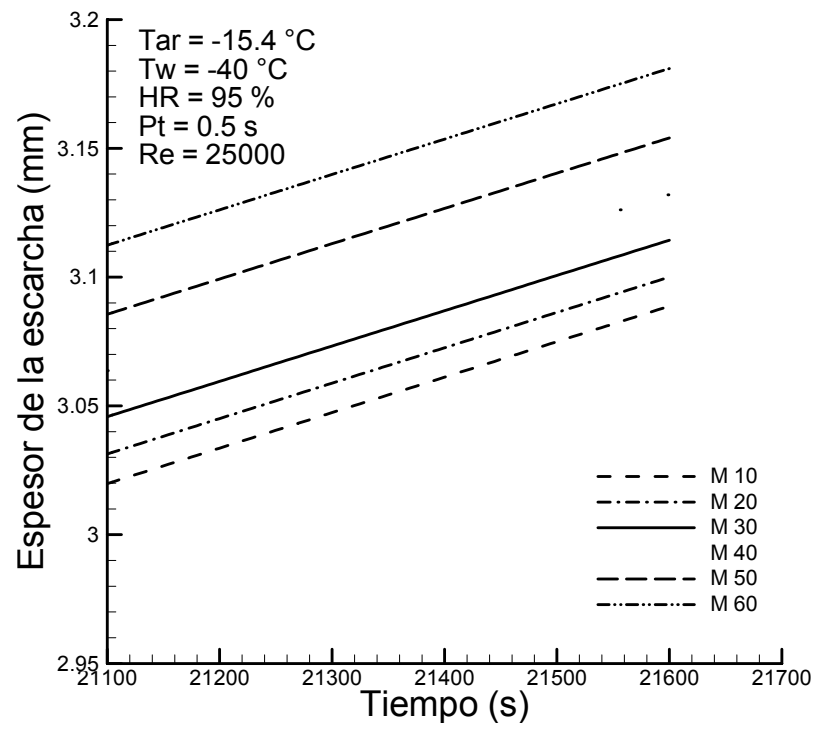

Fig. 4b: Ampliación para cierto Reynolds

Información Tecnológica Vol. - 20 Nº 2 - 2009 
Fracción volumétrica del hielo en la escarcha $\left(\varepsilon_{0} \circ E_{0}\right)$ : En los resultados encontrados en la literatura, se dá poca importancia a la influencia de la condición inicial para el cálculo de la fracción volumétrica del hielo. La mayoría evita describir la fase de cristalización de la escarcha, asignándole un valor inicial. Experimentalmente, dicha fase resulta de espesor insignificante, durando sólo segundos.

Los gráficos, Fig. 5 y Fig. 6, se obtienen para 2 hrs. de simulación para condiciones de operación típicas de congelador definidas por: Tar $=-15.4{ }^{\circ} \mathrm{C}, \mathrm{Tw}=-40{ }^{\circ} \mathrm{C}, \mathrm{HR}=95 \%, \operatorname{Re}=10000$, empleando valores seleccionados $(\mathrm{Pt}=0.5 \mathrm{~s}$ y $\mathrm{M}=30)$. Los datos se generan para el tiempo final y/o para la superficie de la escarcha.

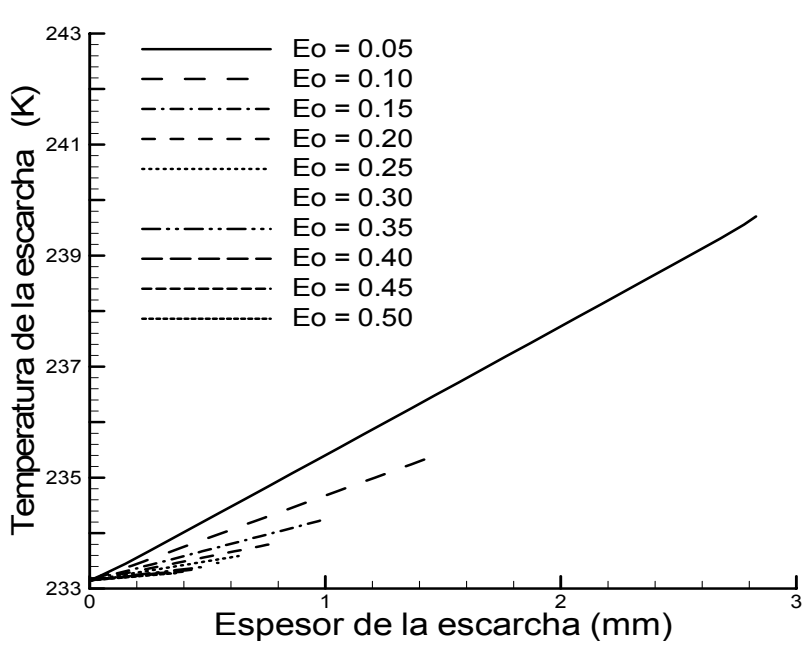

Fig. 5: Efecto de $\varepsilon_{\mathrm{o}}\left(\mathrm{E}_{0}\right)$ en la temperatura

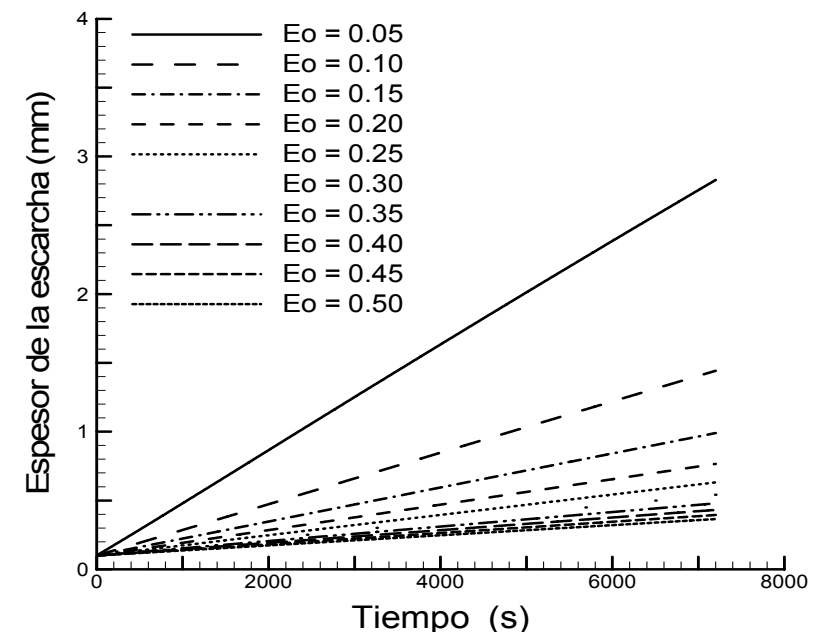

Fig. 6: Efecto de $\varepsilon_{0}\left(E_{0}\right)$ en el espesor

Valores de $\varepsilon_{0}$ elevados, entre 0,20 y 0,5 , provocan menos alteración en los resultados. Se elige un valor de $\varepsilon_{0}=0.25$. Chen et al. (1999) utiliza $\varepsilon_{0}=0.3$, pero similar a este trabajo no verifica diferencias significativas para valores entre 0.3 y 0.6 .

\section{Validación del programa de simulación}

Con este objeto, el código computacional se aplicó, Figs. 7 y 8, para las condiciones definidas en la obtención de los resultados experimentales, presentes en la literatura.

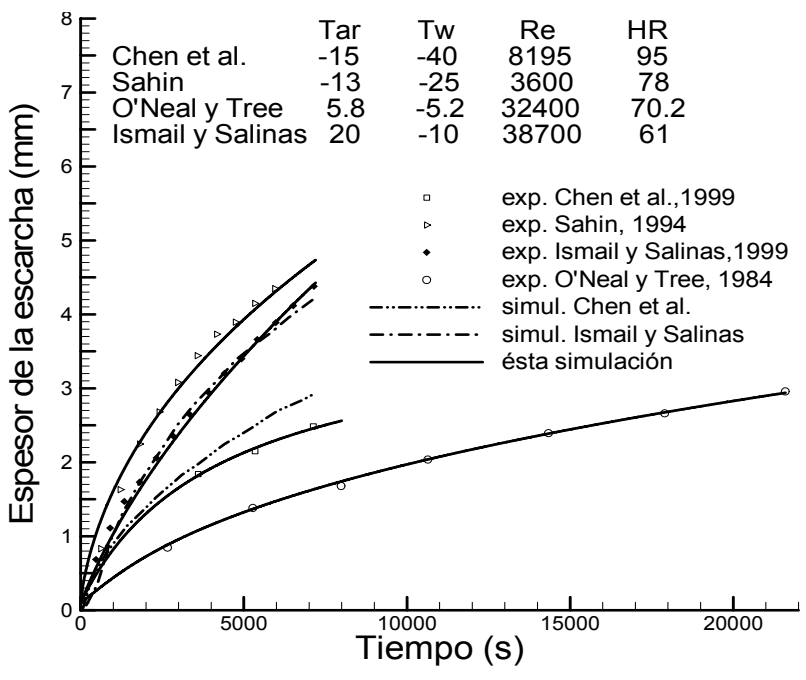

Fig. 7: Simulación vs. varios autores

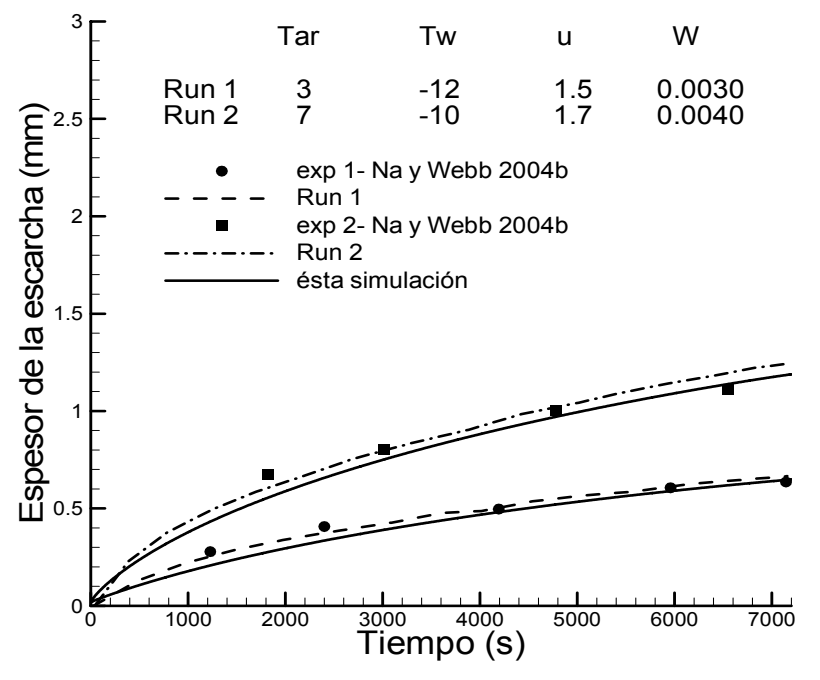

Fig. 8: Simulación vs. Na y Webb (2004b)

Se observa mejor concordancia, Fig. 7, de esta simulación que la mostrada por métodos numéricos de Ismail y Salinas (1999) y de Chen et al. (1999) en relación a los datos experimentales en común, e incluso de otros autores. La concordancia no es tan buena, Fig. 8, cuando comparada con métodos numéricos de sobresaturación (Run1 y Run2) y datos experimentales, ambos de Na y Webb (2004b). 


\section{RESULTADOS Y DISCUSIÓN}

El uso del código computacional, en FORTRAN 77, considerando $\mathrm{Pt}=0.5 \mathrm{~s}, \mathrm{M}=30$ y $\varepsilon_{0}=0.25$ permiten generar los gráficos, con condiciones operacionales indicadas.

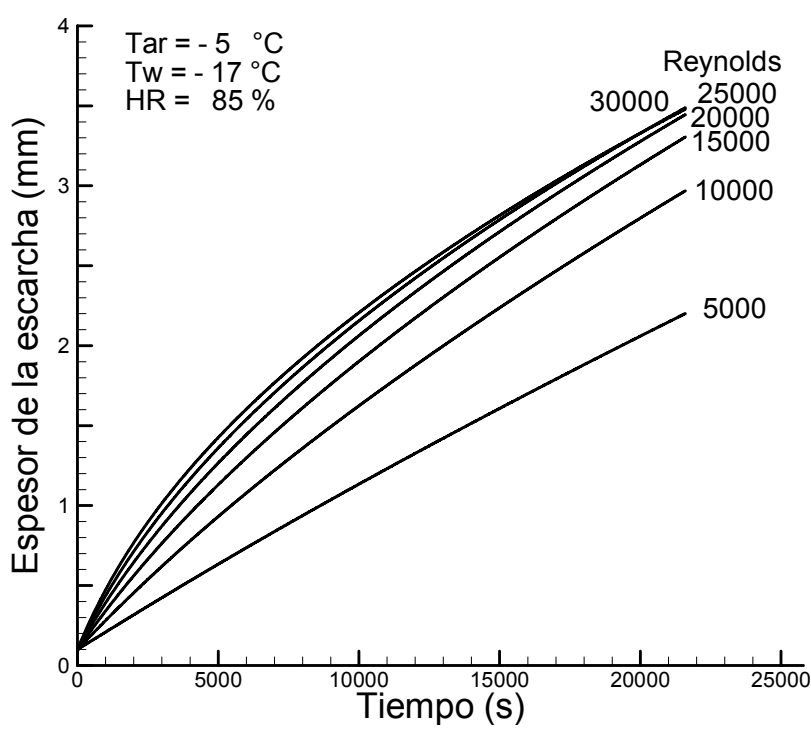

Fig. 9: Crecimiento de la escarcha en el tiempo para distintos Reynolds

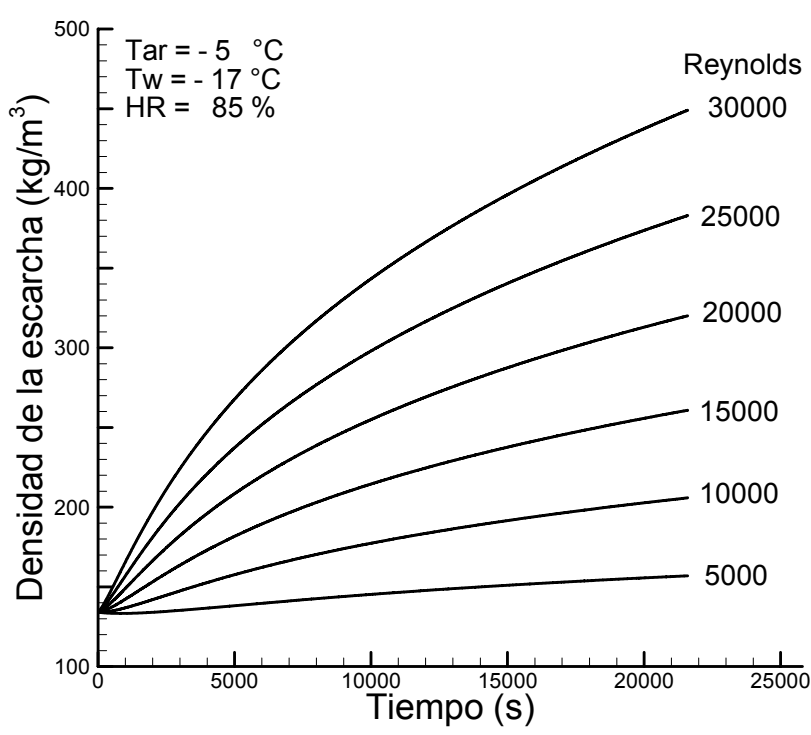

Fig. 11: Variación de densidad de la escarcha en el tiempo con el $\mathrm{N}^{\circ}$ de Reynolds

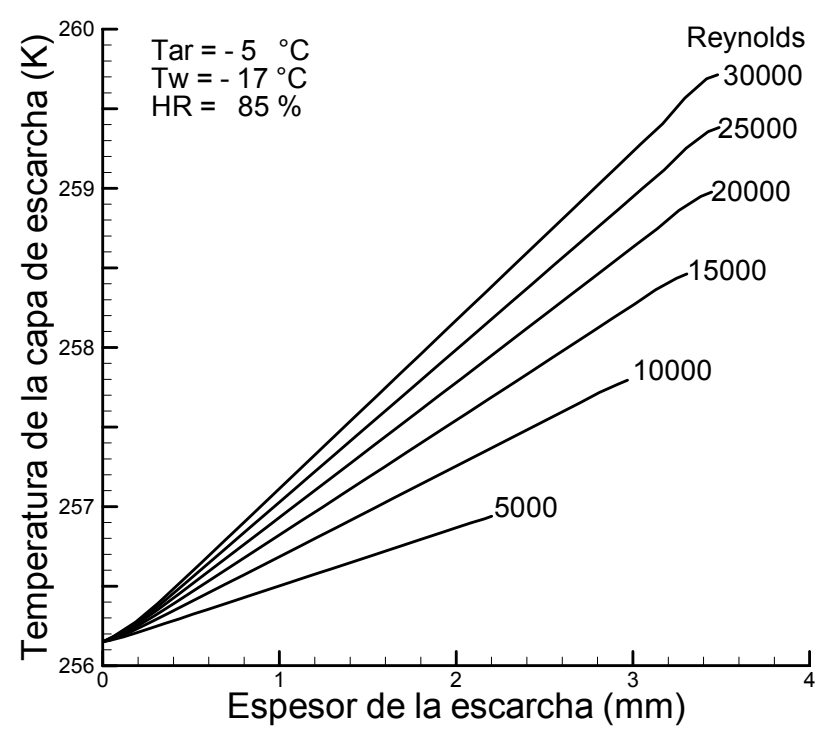

Fig. 10: Distribución de temperaturas en capa de escarcha, para distintos Reynolds.

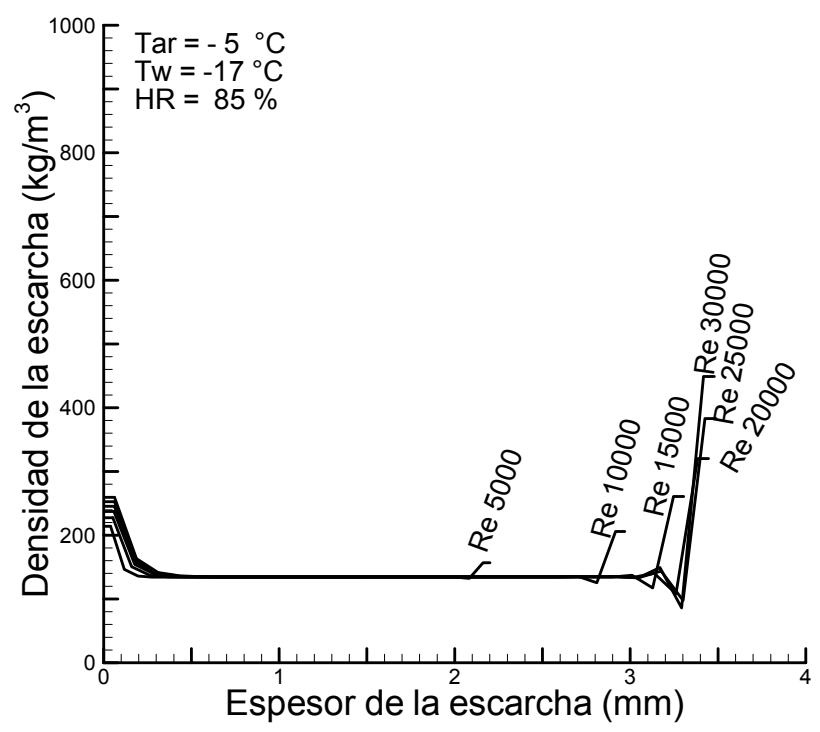

Fig. 12: Variación de densidad de la escarcha en el espesor, con el $\mathrm{N}^{\circ}$ de Reynolds

La variación en el espesor de la capa de escarcha, Fig. 9, para un tiempo de simulación de 6 horas, muestra que a mayores Reynolds, sus efectos en el espesor tienden a reducirse. Esto corrobora comentarios de O'Neal y Tree (1984) relativos a un Reynolds límite de 15900. Números de Reynolds elevados, Fig. 10, favorecen la difusión de vapor de agua, incrementando la temperatura superficial por la mayor resistencia térmica. La Fig. 11 verifica la influencia del Reynolds en la densidad de la escarcha, a causa de variaciones en coeficientes de transferencia de calor y análogamente de transferencia de masa. La densidad de la escarcha, Fig. 12, se observa bastante coherente excepto en su superficie. Esto se explica fundamentalmente porque el contorno de la escarcha se analiza diferente a sus puntos interiores, pues la expresión de conservación del vapor está alterada en estas zonas. Una comparación entre Figs. 13 y 11, permite apreciar comportamiento similar, esto reafirma la existencia de una correlación entre la densidad y conductividad de la escarcha. Correlaciones entre estas propiedades son usadas por varios autores, entre ellos Lee et al. (1997). 


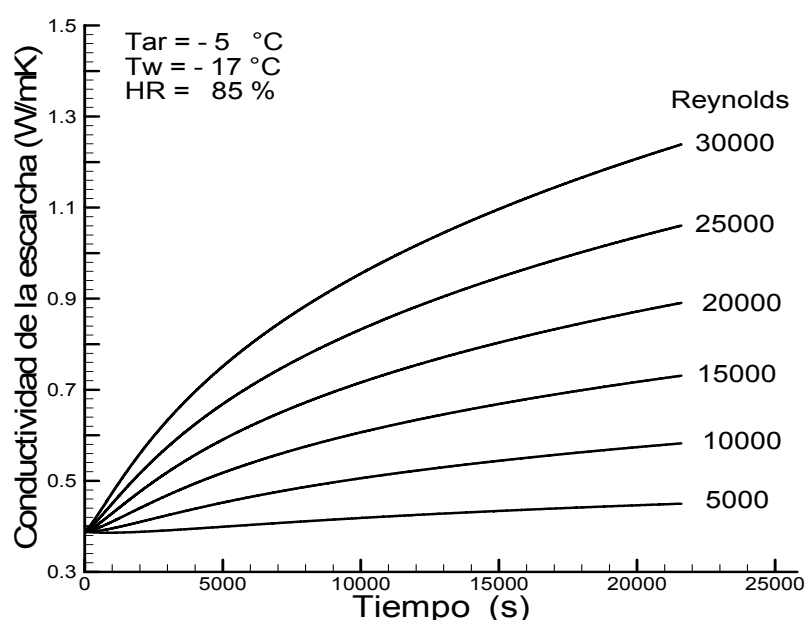

Fig. 13: Conductividad de la escarcha en el tiempo para Reynolds distintos

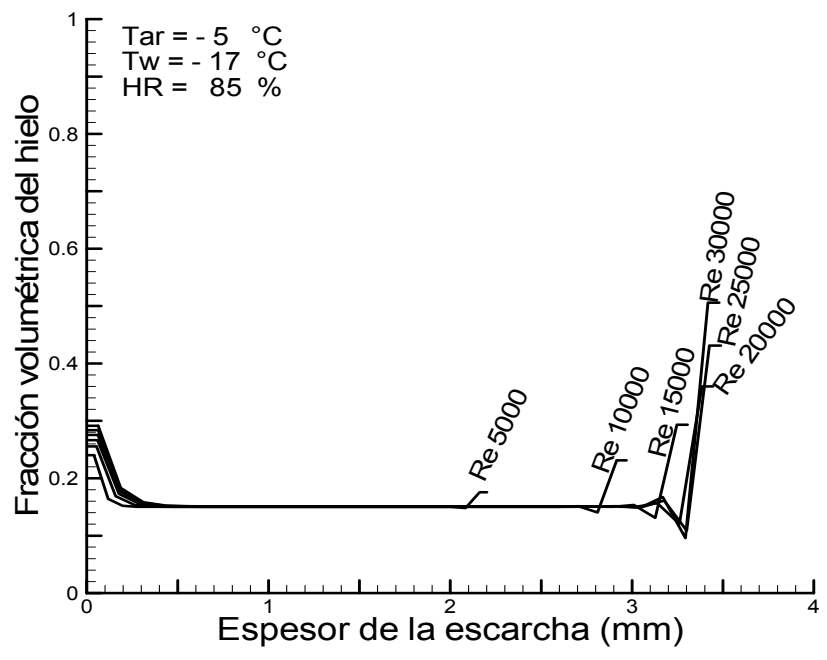

Fig. 15: Fracción volumétrica del hielo en la capa de escarcha para Reynolds distintos.

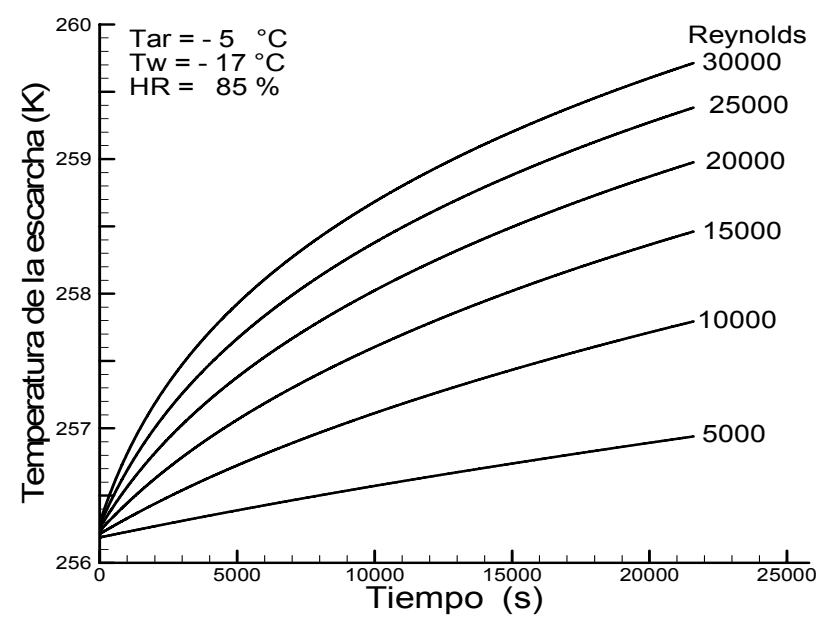

Fig. 14: Temperatura de la escarcha en el tiempo para Reynolds distintos

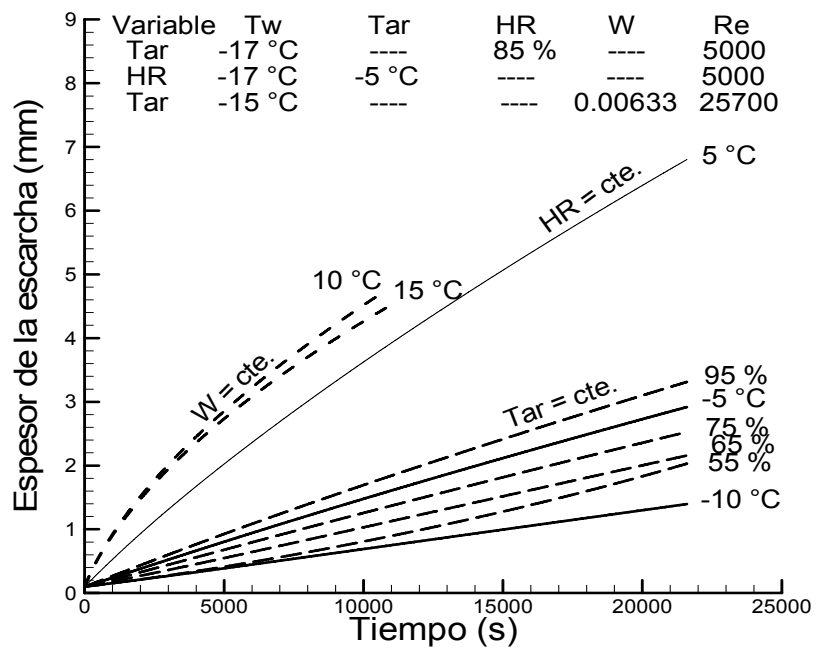

Fig. 16: Efecto de Tar, HR y W en el espesor de la escarcha

La temperatura crece más rápidamente, Fig. 14, a mayores Reynolds. La fracción volumétrica del hielo, considerada complemento de la porosidad de la escarcha, Fig. 15, se comporta opuesto a ésta y similar a la densidad. Resulta difícil encontrar información experimental y/o teórica relativa a esta propiedad de la escarcha. Luer y Beer (2000) muestran una distribución de porosidad asumida en la superficie de la escarcha, para un Reynolds de 1500, de aproximadamente $95 \%$. En este trabajo se logra porosidad promedio de $80 \%$ (fracción volumétrica $20 \%$ ), con nula influencia del Reynolds. El comportamiento en la superficie se explica en forma similar al de la densidad.

Incrementos de temperatura (a HR constante) o de la humedad relativa del aire (a $\mathrm{T}_{\mathrm{ar}}$ constante), Fig. 16, provocan aumentos en el espesor de la escarcha formada. En el primer caso, $\mathrm{T}_{\mathrm{ar}}$ crece de $-10{ }^{\circ} \mathrm{C}$ a $5{ }^{\circ} \mathrm{C}$ y va acompañada de aumentos en su humedad específica (W), por lo cual es mayor la cantidad de vapor de agua que difunde y ablima a la forma de escarcha. En el segundo caso HR varía (y paralelamente $\mathrm{W}$ ) entre $55 \%$ y $95 \%$, provocando similarmente un aumento en el potencial de transporte entre el aire y la superficie de la escarcha (Lee et al. 1997), facilitando la formación de una capa espesa,. Por último, el espesor de escarcha se reduce a mayor temperatura del aire, cuando varía (a W constante) de 10 a $15{ }^{\circ} \mathrm{C}$. Una explicación de esto puede ser que, a causa del mayor gradiente de temperaturas provocado con temperaturas elevadas, las moléculas de vapor que llegan a la superficie de escarcha no tienen tiempo suficiente para formar las estructuras cristalinas adecuadas, afectando así el crecimiento de la escarcha. Esto último concuerda con estudios teóricos de Yang et al. (2006) y experimentales de Sahin (1994). 


\section{CONCLUSIONES}

Fue presentado un modelo físico para simular el crecimiento y densificación de la capa de escarcha, formada por el depósito de vapor de agua procedente del aire húmedo, cuando es sometido a un proceso de transferencia de calor y masa por contacto, con una superficie plana suficientemente fría. Este simulador muestra la evolución en el tiempo y en el espacio, de la densidad, espesor, conductividad térmica efectiva, calor específico, y porosidad de la escarcha. Este modelo, muestra fuerte concordancia con datos experimentales y mejor que la presentada por otros autores. Leves diferencias entre el modelo de saturación y el de sobresaturación favorecen a este último. Los resultados del modelo permiten verificar que el espesor de la escarcha, que es posiblemente la única magnitud medible con exactitud, es influido directamente por el número de Reynolds, sin embargo se insensibiliza para altos valores de éste. La densidad de la escarcha se incrementa en el tiempo para números de Reynolds mayores, a causa de las variaciones en el coeficiente de transferencia de calor. Esta propiedad muestra gran similitud de comportamiento con la conductividad térmica reforzando la existencia de una correlación entre ellas. La humedad relativa del aire tiene efecto directo sobre el espesor de la escarcha explicado por el aumento en el potencial de transporte entre el aire y la superficie de ésta. La formación de escarcha es afectada inversa o directamente por la temperatura del aire, dependiendo de si la humedad específica permanece o no constante, respectivamente. El número de Reynolds tiene pobre o nula influencia en la fracción volumétrica de hielo en la capa de escarcha. Esta distribución es muy difícil de medir y dada la importancia práctica de este resultado, posee gran relevancia para posteriores estudios de adhesión y remoción de escarcha; Se observa lo mismo sobre densidad interna de la escarcha. La fracción volumétrica inicial influye inversamente en el espesor y la temperatura y directamente en densidad de la escarcha.

En general se ha desarrollado, por el método de diferencias finitas, un código computacional robusto que permite simular la formación de escarcha sobre una placa fría obteniendo información temporal y espacial de sus propiedades termofísicas.

\section{NOMENCLATURA}

$\mathrm{Cp}=$ calor específico (presión cte) $\left[\mathrm{J} /\left(\mathrm{kg}^{\circ} \mathrm{C}\right)\right]$

$D_{A B}=$ Coeficiente de difusión de masa, $\left(\mathrm{m}^{2} / \mathrm{s}\right)$

$D_{\text {eff }}=$ Coeficiente de difusión efectiva $\left(\mathrm{m}^{2} / \mathrm{s}\right)$

$\mathrm{F}=$ factor de corrección del parámetro de resistencia a la difusión

$\mathrm{h}=$ Coeficiente de transferencia

$\mathrm{HR}=$ humedad relativa del aire $(\%)$

$\mathrm{I}_{\text {ig }} \quad=$ calor latente de sublimación $(\mathrm{J} / \mathrm{kg})$

$\mathrm{k}=$ conductividad térmica $[\mathrm{W} /(\mathrm{mK})]$

$\mathrm{k}_{\text {eff }}=$ conductividad térmica efectiva de la escarcha $[\mathrm{W} /(\mathrm{mK})]$

$\mathrm{L} \quad=$ longitud característica de la placa

$\mathrm{Nu}=$ número de Nusselt

$\mathrm{p}_{\mathrm{atm}}=$ presión atmosférica $(\mathrm{Pa})$

$\operatorname{Pr}=$ número de Prandtl

$\mathrm{t}=$ tiempo (s)

$\mathrm{T}=$ temperatura de la escarcha $(\mathrm{K})$

$\mathrm{T}_{\mathrm{ar}}=$ temperatura media del aire $\left({ }^{\circ} \mathrm{C}\right)$

$\mathrm{T}_{\mathrm{w}}=$ temperatura de la placa fría $\left({ }^{\circ} \mathrm{C}\right)$

$\mathrm{u}=$ velocidad media del aire $(\mathrm{m} / \mathrm{s})$

$\mathrm{W}$ = humedad específica aire húmedo $(\mathrm{kg} / \mathrm{kg})$

$\mathrm{y} \quad=$ eje de crecimiento de la escarcha $(\mathrm{m})$ $\mathrm{y}_{\mathrm{s}} \quad=\quad$ espesor de la escarcha $(\mathrm{m})$

Símbolos griegos

$\varepsilon=$ fracción volumétrica del hielo en la escarcha

$\varepsilon_{\mathrm{g}} \quad=$ fracción volumétrica del gas en la escarcha

$\rho=$ densidad (de la escarcha) $\left(\mathrm{kg} / \mathrm{m}^{3}\right)$.

$\rho_{\mathrm{a}} \quad=$ densidad del aire seco $\left(\mathrm{kg} / \mathrm{m}^{3}\right)$

$\rho_{\mathrm{l}} \quad=$ densidad del hielo $\left(\mathrm{kg} / \mathrm{m}^{3}\right)$.

$\rho_{\mathrm{p}} \quad=$ densidad en el poro

$\rho_{v}=$ densidad del vapor en el aire húmedo $\left(\mathrm{kg} / \mathrm{m}^{3}\right)$. porosidad de la escarcha

Subíndices

$\infty=$ en zona no perturbada

a $=$ del aire seco

$\mathrm{CV} \quad=$ convectivo de calor

$\mathrm{g}=$ del gas (aire húmedo)

$\mathrm{I}=$ del hielo

$\mathrm{m} \quad=$ convectivo de masa

o $\quad=$ inicial

$\mathrm{s}=$ en superficie de la escarcha

$\mathrm{V} \quad=$ del vapor de agua 


\section{AGRADECIMIENTOS}

A la Universidad del Bío-Bío Chile, por el soporte financiero a través del proyecto 041513 3/RS, y NCL., reconoce el apoyo de CNPq.

\section{REFERENCIAS}

ASHRAE, "Psychrometrics". Fundamentals Handbook, Cap. 6. , (1997).

Auracher, H.; "Water vapor diffusion and frost formation in capillaries". Annex 1972 -1. In. Bulletin of the International Institute of Refrigeration. Communications B1, B2 and E1, Freudenstadt, p. 477-488 (1972).

Chen, H., L. Thomas y R. Besant; "Modeling frost characteristics on heat exchanger fins: Part II, Model validation and limitations", Ashrae Transactions: 106, part. 2, 1-9 (2000).

Chen, H., R. Besant y Y. Tao; "Frost characteristics and heat transfer on a flat plate under freezer operating conditions: Part II, Numerical modeling and comparison with data", Ashrae Transactions: 105, part. 2, 252-259 (1999).

Dietenberger, M.; "Generalized correlation of the water frost thermal conductivity", Int. J. of Heat and Mass Transfer: 26 (4), 607-619 (1983).

Gatica, J.A.; "Um modelo para crescimento da geada”. Tese de doutorado. PEM/COPPE/ UFRJ. Rio de Janeiro, Brasil (2003).

Ismail, K.A.R. y C.S. Salinas; "Modeling of frost formation over parallel cold plates", International Journal of Refrigeration: 22, 425-441 (1999).

Kim, J.S., D.K. Yang. y K.S. Lee; "Dimensionless correlations of frost properties on a cold cylinder surface", Int. Journal of Heat and Mass Transfer: In press, corrected proof (2008).

Lee, K.S., W.S. Kim y T.H. Lee; "A one-dimensional model for frost formation on a cold flat surface", International Journal of Heat and Mass Transfer: 40(18), 4359-4365 (1997).

Lee, Y.B. y S.T. Ro; "Analysis of the frost growth on a flat plate by simple models of saturation and supersaturation", Experimental Thermal and Fluid Science: 29, 685-696 (2005).

Le Gall, R., J.M. Grillot y C. Jallut; "Modeling of frost growth and densification", Int. J. of Heat and Mass Transfer: 40 (13), 3177-3187 (1997).

Luer, A. y H. Beer; "Frost deposition in parallel plate channel under laminar flow conditions", Int. J. Thermal Science: 39, 85-95 (2000).

Mao, Y., R.W. Besant y K.S. Rezkallah; "Measurement and correlations of frost properties with airflow over a flat plate", Ashrae Transactions: 98 (Part 2), 65-78 (1992).

Mao, Y., R.W. Besant y H. Chen; "Frost characteristic and heat transfer on a flat plate under freezer operating conditions: Part I, Experimentation and Correlations", Ashrae Transactions: 99 (1), 739-745 (1999).

$\mathrm{Na}, \mathrm{B}$. y R.L. Webb; "Mass transfer on and whitin a frost layer", Int. J. Heat Mass Transfer: 47 (5), 899-911 (2004a).

Na, B. y R.L. Webb; "New model for frost growth rate", Int. J. Heat Mass Transfer: 47 (5), 925-936 (2004b). 
O'Neal, D.L. y D.R. Tree; "Measurement of frost growth and density in a parallel plate geometry", Ashrae Transactions: 90, part. 2, 278-290 (1984).

O’Neal, D.L. y D.R. Tree; “A review of frost formation in simple geometries”, Ashrae Transactions: 91, part. 2A, 267-281 (1985).

Padki, M.M., S.A. Sherif y R.M. Nelson; "A simple method for modeling the frost formation phenomenon in different geometries", Ashrae Transactions: 95, 1127-1137 (1989).

Qu, K., K. Satoru y J. Yi; "Local variation of frost layer thickness and morphology", Int. J. Thermal Sciences: 45 (2), 116-123 (2006).

Sahin, A.Z.; "An experimental study on the initiation and growth of frost formation on a horizontal plate”, Experimental Heat Transfer: 7, 101-119 (1994).

Tao, Y.X., R.W. Besant, y K.S. Rezkallah; “A mathematical model for predicting the densification and growth of frost on a flat plate", Int. J. Heat Mass Transfer: 36 (2), 353-363 (1993).

Tudor, V., M.M. Ohadi y F.H.R. França; "An experimental investigation on frost control using DC and AC electric fields on horizontal, down ward -facing plate". HVAC\&R Research: 9, Part. 2, 203213 (2003).

Yang, D.K., K.S. Lee y D.J. Cha; "Frost formation on a cold surface under turbulent flow”. Int. Journal of Refrigeration: 29, 164 -169 (2006).

Yun, R., Y. Kim y M. Min; "Modeling of frost growth and frost properties with airflow over a flat plate". Int. Journal of Refrigeration: 25, 362-371 (2002). 